\title{
Efeitos da oxigenoterapia hiperbárica em ratos submetidos à ligadura das veias hepáticas: avaliação da mortalidade e da histologia do fígado e baço ${ }^{1}$
}

\author{
Hyperbaric oxygen therapy in rats submitted to hepatic veins ligation: mortality \\ valuation and histological study of liver and spleen
}

\author{
Ricardo Costa-Val' ${ }^{2}$, Tarcizo Afonso Nunes ${ }^{3}$, Roberto Carlos de Oliveira e Silva ${ }^{4}$, Tatiana Karina De Puy e Souza \\ 1. Resumo Tese de Doutorado, Programa de Pós-Graduação em Cirurgia da Faculdade de Medicina da Universidade de Minas Gerais (UFMG). \\ 2. Mestre e Doutor em Cirurgia pela UFMG. Professor da Graduação e Pós - Graduação da Universidade Vale do Rio Verde - UNINCOR. \\ 3. Prof. Adjunto-Doutor do Departamento de Cirurgia da Faculdade de Medicina da UFMG. \\ 4. Mestre e Doutor em Cirurgia pela UFMG. Professor substituto da Faculdade de Medicina da UFMG. \\ 5. Acadêmica da Faculdade de Medicina de Barbacena/MG - UNIPAC.
}

\begin{abstract}
RESUMO
Objetivo: Avaliar os efeitos da oxigenoterapia hiperbárica em ratos submetidos a ligadura das veias hepáticas quanto a mortalidade dos animais e alterações na histologia do fígado e baço. Métodos: Foram utilizados 30 animais machos adultos da espécie Holtzman, distribuídos aleatoriamente, em dois grupos de 15 animais cada, assim designados: grupo 1 - ligadura das veias hepáticas; grupo 2 - ligadura das veias hepáticas associada à oxigenoterapia hiperbárica. Todos os animais foram submetidos à anestesia geral por meio de solução contendo cloridrato de cetamina $(40 \mathrm{mg} / \mathrm{ml})$ e cloridrato de meperidina $(10 \mathrm{mg} / \mathrm{ml})$ na dose de $50 \mathrm{mg} / \mathrm{Kg} /$ peso, laparotomia mediana e ligadura das veias hepáticas. A oxigenoterapia hiperbárica foi aplicada nos animais do grupo 2, a partir da oitava hora do pós-operatório, por 120 minutos, sendo 90 minutos sob pressão de 2,5 atmosferas e 15 minutos no início e final da terapêutica, para promover a compressão e descompressão gradativa no período de 20 dias consecutivos. No $21^{\circ}$ dia de pós-operatório, os animais foram mortos por inalação de éter, submetidos à laparotomia e extirpação dos fígados e baços para exame histológico. Foi comparada a evolução dos animais dos dois grupos quanto à mortalidade e histologia do fígado e do baço, aplicando-se o teste exato de Fisher, considerando-se a diferença significante o valor de $p<0,05$. Resultados: Ocorreram sete (46,67\%) mortes nos animais do grupo 1 e nenhuma morte nos animais do grupo 2. Os exames histológicos dos fígados e baços dos animais dos grupos 1 e 2 mostraram as seguintes alterações: trombose nas veias hepática, porta e centro-lobular estavam presentes em cinco $(33,3 \%)$ animais do grupo 1 e ausente no grupo 2; necrose dos hepatócitos caracterizada como acentuada em sete $(46,7 \%)$ e como leve em oito $(53,3 \%)$ animais do grupo 1 , enquanto em todos os animais do grupo 2 esta alteração foi caracterizada como leve; presença de células de Kupffer muito proeminentes e hipertrofiadas em 14 (93,3\%) animais do grupo 1 e pouco proeminentes e acentuadas todos os animais do grupo 2; congestão da polpa vermelha considerada acentuada em seis (40\%) e moderada em nove (60\%) dos animais do grupo 1 e em todos os animais do grupo 2; hemossiderinose moderada ou acentuada em 14 animais $(93,3 \%)$ do grupo 1 e leve em todos os animais do grupo 2 . A comparação entre os dois grupos mostrou diferença significativa em todas a variáveis estudadas $(p<0,05)$. Conclusões: A oxigenoterapia hiperbárica em ratos submetidos à ligadura das veias hepáticas reduziu a incidência de mortes pós-operatórias e atenuou os efeitos deletérios e precoces sobre o fígado e o baço.
\end{abstract}

Descritores: Oxigenoterapia. Ratos. Histologia. Fígado. Baço. Ratos.

\begin{abstract}
Purpose: To evaluate the effects of hyperbaric oxygen therapy in rats submitted to instantaneously hepatic vein obstruction. Methods: 30 Holtzman adult male rats were utilised, distributed into two groups: 1) hepatic vein obstruction; 2) hepatic vein obstruction associated with hyperbaric oxygen therapy. General anaesthesia was utilized by a solution composed of ketamine chloride $(40 \mathrm{mg} / \mathrm{ml})$ and meperidine chloride $(10 \mathrm{mg} / \mathrm{ml})$ in a dose of $50 / \mathrm{mg} / \mathrm{weight}$, applied into the right gluteus muscle. The animals belonged to group 2 were submitted to hyperbaric oxygen therapy, 8 hours after the operations, in a 2,5 atmosphere, which lasts 120 minutes per day, in consecutive 20 days. The statistical analysis was made in relation to mortality and histological study of livers and spleens utilizing the Fisher test, and the results were considered statistically significant when $p<0,05$. Results: Occurred seven (46,67\%) deaths between animals belonged to group 1 and no deaths in the animals belonged to group 2. The histological studies made in the livers and spleens of the animals belonged to group 1 showed many alterations in the following percentages: thrombosis of hepatic, portal and center-lobular veins in five (33,3\%), very extensive necrosis of liver cells in seven (46,7\%), and light in eight (53,3\%), Kupffer cells developed and hypertrophied in $14(93,3 \%)$, high congestion of the spleen purple in six (40,0\%) and moderate and severe hemossiderinosis spleen in $14(93,3 \%)$. The analysis of this parameters in the group 2 only showed light necrosis of liver cells, Kupffer cells
\end{abstract}


light developed and hypertrophied, moderated congestion of the spleen purple and light hemossiderinosis spleen. All these parameters analysed showed significantly difference $(p<0,05)$ between these two groups. Conclusions: It could be concluded that the hyperbaric oxygen therapy applied in rats, with instantaneously hepatic vein obstruction decreased their post-surgical mortality and their early deleterious effects in the liver and spleen

Key words: Oxygen Inhalation Therapy. Histology. Liver. Spleen. Rats.

\section{Introdução}

A síndrome de hipertensão porta caracteriza-se pela hepatoesplenomegalia, circulação colateral hepatofuga e ascite. É freqüente em todo o mundo, mas sua etiologia varia nas diferentes regiões, até mesmo dentro de um mesmo país. Suas principais complicações são a hemorragia digestiva alta e insuficiência hepática, cujas causas mais comuns são a cirrose e a esquistossomose, sendo as obstruções venosas mais raras e mais graves, sobretudo quando ocorre nas veias hepáticas ${ }^{(1)}$. A síndrome de BuddChiari decorre da obstrução primária ou secundária da drenagem venosa do fígado, devido a várias etiologias. Suas manifestações clínicas podem ocorrer de maneira aguda ou crônica e as alterações nas provas das funções hepáticas são mais evidentes nos eventos agudos e seu diagnóstico definitivo é feito por métodos de imagem e pela histologia do fígado. $\mathrm{Na}$ abordagem terapêutica são empregados agentes farmacológicos, derivações portassistêmicas (intrahepáticas transjugulares, portacava e mesentéricocava através de próteses) e dispositivos endovasculares. $\mathrm{O}$ transplante hepático ortotópico é o tratamento definitivo nos casos mais graves ou de difícil controle por outras medidas. Mas, esse procedimento é complexo e não é possível empregá-lo em todos os pacientes, em decorrência dos vasos comprometidos ou por falta de órgãos e serviços especializados, o que resulta em longo tempo de espera. Em conseqüência, muitos pacientes com insuficiência hepática grave não sobrevivem até o momento do transplante ${ }^{(2-5)}$. É relevante, portanto, pesquisar novos métodos terapêuticos capazes de amenizar ou combater os efeitos da obstrução das veias hepáticas sobre o fígado, oferecendo aos doentes a possibilidade de melhoria das condições clínicas e aguardar o tratamento definitivo. A oxigenoterapia hiperbárica tem se mostrado capaz de melhorar a evolução de animais e seres humanos com diversas afecções nas quais existe, em suas fisiopatologias, a tríade: hipóxia, isquemia e reperfusão ${ }^{(6-9)}$. Sabe-se que a congestão venosa grave pode induzir isquemia celular, principalmente quando ocorre de forma súbita ${ }^{(10,11)}$. A aplicabilidade e indicação da oxigenoterapia hiperbárica estão asseguradas cientificamente para várias doenças ${ }^{(7,12,13)}$, mas existe vasto campo da área médica no qual sua eficácia é desconhecida e são necessários estudos experimentais e clínicos. Seguindo essa linha de raciocínio, realizou-se esta pesquisa com o objetivo de avaliar os efeitos da oxigenoterapia hiperbárica em ratos submetidos a congestão hepática por meio da ligadura das veias hepáticas.

\section{Métodos}

Este estudo seguiu os princípios éticos na experimentação animal descritos pelo COBEA - Colégio
Brasileiro de Experimentação Animal e foi aprovado pela Câmara do Departamento de Cirurgia da Faculdade de Medicina da Universidade Federal de Minas Gerais. Para a realização do experimento foram empregados 30 ratos machos Holtzman, que permaneceram em gaiolas apropriadas, expostos à luz em ciclos regulares de 12 horas e receberam ração industrial (Nuvilab $\mathrm{CR}_{1}{ }^{\circledR}$, Laboratório Nuvital) e água ad libidum em todas as fases do estudo. Os animais foram distribuídos, aleatoriamente e por sorteio, em dois grupos:

- Grupo 1 - ligadura das veias hepáticas: 15 animais submetidos à congestão hepática induzida por meio da ligadura das veias hepáticas;

- Grupo 2 - ligadura das veias hepáticas associada à oxigenoterapia hiperbárica: 15 animais submetidos à congestão hepática induzida por meio da ligadura das veias hepáticas e à oxigenoterapia hiperbárica;

\section{Procedimento cirúrgico}

Os ratos foram submetidos a anestesia geral mediante injeção no músculo glúteo superficial direito da solução de $8 \mathrm{ml}$ de cloridrato de cetamina $(40 \mathrm{mg} / \mathrm{ml})$ e $2 \mathrm{ml}$ de cloridrato de meperidina $(10 \mathrm{mg} / \mathrm{ml})$, na dose de $50 \mathrm{mg} / \mathrm{Kg} / \mathrm{peso}$, referente ao cloridrato de cetamina. Realizou-se a tonsura dos pêlos do tórax e abdômen e a anti-sepsia com solução alcoólica de polivinilpirrolidona-iodo a 1,0\%. O procedimento cirúrgico iniciou-se pela laparotomia mediana, que se estendeu a partir do terço inferior do apêndice xifóide até o púbis, seguido de exploração da cavidade abdominal, dissecção delicada do ligamento redondo do fígado e identificação das veias hepáticas. Essas veias foram dissecadas e ligadas, em conjunto, com fio de seda trançada e siliconizada 4-0. A seguir, foram feitas a revisão da hemostasia e a síntese da cavidade abdominal em duas camadas de sutura contínua com fio de nylon 3-0.

\section{Pós-operatório}

Concluídas as operações, os animais foram alojados em gaiolas individuais até a recuperação anestésica e em torno da quarta hora foram agrupados em gaiolas coletivas e examinados em intervalos regulares de cerca de 4 horas até o término do experimento. Foram excluídos do estudo os animais que morreram imediatamente após a ligadura das veias hepáticas devido a exsangüinação, lesão iatrogênica do fígado, pneumotórax e parada cardio-respiratória ou que não apresentaram boa recuperação cirúrgico-anestésica nas primeiras oito horas de pós-operatório. O número de animais utilizados a fim de se obter o total de 15 em cada grupo foi de 25 e 22, para os grupos 1 e 2 , respectivamente. 


\section{Oxigenoterapia hiperbárica}

Empregou-se câmara hiperbárica tipo monopaciente, (modelo BLKS 303 MK, Moscou), aprovada para uso no Brasil pela Agência Nacional de Vigilância Sanitária (ANVISA). A oxigenoterapia hiperbárica nos animais do grupo 2 foi aplicada em uma sessão diária durante 20 dias consecutivos, em intervalos regulares de 24 horas, que iniciou na oitava hora após o término das operações nos animais. Foi administrado oxigênio a 100\% com duração total de 120 minutos cada sessão, sendo 90 minutos sob pressão de 2,5 atmosferas e os 15 minutos iniciais e finais para compressão e descompressão gradativas, respectivamente.

\section{Morte dos animais e retirada dos órgãos}

Os fígados e baços dos animais dos dois grupos foram extirpados, fixados em formol a $10 \%$ para estudo microscópico. Aqueles que morreram no decorrer do experimento, a partir da oitava hora do pós-operatório, esse procedimento era realizado logo após a constatação do óbito, para evitar as alterações degenerativas dos órgãos. Os animais que permaneceram vivos foram submetidos à eutanásia por inalação de éter para a extirpação do fígado e baço de acordo com a padronização estabelecida:

- grupo $1-21^{\circ}$ dia de pós-operatório;

- grupo $2-21^{\circ}$ dia de pós-operatório, após as 20 sessões de oxigenoterapia hiperbárica.

\section{Histologia}

Fígados e baços foram processados em blocos de parafina, submetidos a cortes histológicos longitudinais em micrótomo calibrado para 5 micra e corados com hematoxilina e eosina, para estudo à microscopia de luz.

\section{Variáveis estudadas}

A mortalidade foi avaliada nos dois grupos de animais e comparada qualitativamente. Foram pesquisadas alterações histológicas dos fígados e baços, tais como trombose nas veias hepática, porta e centro-lobular, necrose dos hepatócitos, presença de células de Kupffer muito proeminentes e hipertrofiadas, congestão da polpa vermelha e hemossiderose. Compararam-se as alterações histológicas encontradas nos dois grupos de animais pelo teste Exato de Fisher, considerando-se um nível de significância de 5\% $(p<0,05)$.

\section{Resultados}

\section{Mortalidade}

Dentre os 15 animais do grupo 1 , sete $(46,67 \%)$ morreram, sendo cinco $(33,33 \%)$ entre a oitava e $24^{\mathrm{a}}$ horas, um $(6,67 \%)$ no terceiro dia e um $(6,67 \%)$ no sexto dia de pósoperatório. Não ocorreram mortes nos animais do grupo 2.

\section{Histologia hepática dos animais dos grupos 1 e 2}

A trombose nas veias hepática, porta e centro-lobular foi diagnosticada em cinco (33,3\%) dos 15 animais do grupo 1 e em nenhum dos animais do grupo 2. Esse resultado foi significativo $(p<0,05)$ (Tabelas 1 e 2 e Figura 1$)$.

TABELA 1 - Comparação dos animais dos grupos 1 e 2, quanto à presença de trombose nas veias hepáticas, porta e centrolobular.

\begin{tabular}{|c|c|c|c|c|}
\hline \multirow{2}{*}{$\begin{array}{l}\text { Trombose nas veias } \\
\text { hepática, porta e } \\
\text { centro-lobular }\end{array}$} & \multicolumn{2}{|c|}{ Grupo 1} & \multicolumn{2}{|c|}{ Grupo 2} \\
\hline & n & $\%$ & $\mathbf{N}$ & $\%$ \\
\hline Ausente & 10 & 66,7 & 15 & 100,0 \\
\hline Presente & 5 & 33,3 & 0 & 0,0 \\
\hline TOTAL & 15 & 100,0 & 15 & 100,0 \\
\hline
\end{tabular}

Nota: $p$ d" 0,042 (O valor de $p$ refere-se ao teste exato de Fisher)

TABELA 2 - Comparação dos animais dos grupos 1 e 2, quanto à presença de necrose de hepatócitos.

\begin{tabular}{llllll}
\hline Necroce de & \multicolumn{2}{c}{ Grupo 1 } & & \multicolumn{2}{c}{ Grupo 2 } \\
\cline { 2 - 3 } \cline { 5 - 6 } Hepatócitos & $\mathbf{n}$ & \% & & N & \% \\
\hline Leve & 8 & 55,3 & & 15 & 100,0 \\
Acentuada & 7 & 46,7 & & 0 & 0,0 \\
\hline TOTAL & $\mathbf{1 5}$ & $\mathbf{1 0 0 , 0}$ & & $\mathbf{1 5}$ & $\mathbf{1 0 0 , 0}$ \\
\hline
\end{tabular}

Nota: $p=0,006$ ( $\mathrm{O}$ valor de $p$ refere-se ao teste exato de Fisher)

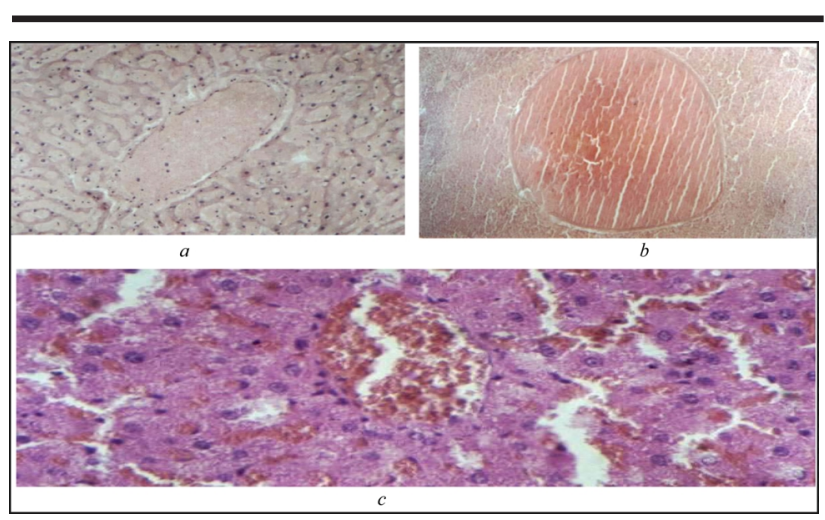

FIGURA 1 - Estudo histológico, por microscopia ótica, dos figados de animais do grupo 1 ( $a$ e $b$ ) e grupo $2(c)$, nos quais se diagnosticaram: trombose da veia hepática $(a)$; trombose centro-lobular com necrose associada (b); estase centrolobular associada à dilatação sinusoidal, além de congestão importante $(c)$. Coloração pela hematoxilinaeosina. Aumentos de 200X ( $a$ e $b$ ) e 400X (c).

A necrose dos hepatócitos apresentou-se acentuada em sete $(46,7 \%)$ e leve em oito $(53,3 \%)$ dos 15 animais do grupo 1. Em todos os animais do grupo 2, essa alteração histopatológica foi considerada leve, revelando diferença significativa entre os dois grupos $(p<0,05)$ (Tabela 3$)$. Observou-se a presença de células de Kupffer muito proeminentes e hipertrofiadas em 14 (93,3\%) dos animais do grupo 1. Em todos do grupo 2 o diagnóstico foi de células de Kupfer pouco proeminentes e hipertrofiadas. Esse resultado mostrou diferença significativa entre os dois grupos $(p<0,05)$ (Tabela 4$)$. 
TABELA 3 - Comparação dos animais dos grupos 1 e 2, quanto à presença de células de Kupffer proeminentes e hipertrofiadas.

\begin{tabular}{llllll}
\hline $\begin{array}{l}\text { Células de Kupffer } \\
\text { Proeminentes e } \\
\text { hipertrofiadas }\end{array}$ & \multicolumn{2}{c}{ Grupo 1 } & & \multicolumn{2}{c}{ Grupo 2 } \\
\cline { 2 - 3 } \cline { 6 - 6 } & $\mathbf{n}$ & $\mathbf{\%}$ & & $\mathbf{n}$ & $\%$ \\
\hline Pouco & 1 & 6,7 & & 15 & 100,0 \\
Muito & 14 & 93,3 & & 0 & 0,0 \\
\hline TOTAL & $\mathbf{1 5}$ & $\mathbf{1 0 0 , 0}$ & & $\mathbf{1 5}$ & $\mathbf{1 0 0 , 0}$ \\
\hline
\end{tabular}

Nota: $p<0,001$ (O valor de $p$ refere-se ao teste exato de Fisher)

TABELA 4 - Comparação dos animais dos grupos 1 e 2, quanto à presença de congestão da polpa vermelha.

\begin{tabular}{llllll}
\hline Congestão da & \multicolumn{2}{c}{ Grupo 1 } & & \multicolumn{2}{c}{ Grupo 2 } \\
\cline { 2 - 3 } \cline { 5 - 6 } polpa vermelha & $\mathbf{n}$ & $\mathbf{\%}$ & & N & \% \\
\hline Moderada & 9 & 60,0 & & 15 & 100,0 \\
Acentuada & 6 & 40,0 & & 0 & 0,0 \\
\hline TOTAL & $\mathbf{1 5}$ & $\mathbf{1 0 0 , 0}$ & $\mathbf{1 5}$ & $\mathbf{1 0 0 , 0}$ \\
\hline
\end{tabular}

Nota: $p=0,017$ ( $\mathrm{O}$ valor de $p$ refere-se ao teste exato de Fisher)

Histologia esplênica dos animais pertencentes aos grupos 1 e 2

Nos animais do grupo 1 foram diagnosticas congestão acentuada da polpa vermelha em seis $(40,0 \%)$ animais e em todos do grupo 2 a congestão da polpa vermelha foi considerada como moderada, mostrando diferença significativa entre os dois grupos $(p<0,05)$ (Tabela 5 e Figura 2). Dos 15 animais do grupo 1, 14 (93,3\%) apresentaram hemossiderose moderada ou acentuada; no grupo 2 essa alteração foi diagnosticada como leve, sendo a diferença significativa $(p<0,05)$ (Figura 2).

TABELA 5 - Comparação dos animais dos grupos 1 e 2, quanto à hemossiderose

\begin{tabular}{llllll}
\hline \multirow{2}{*}{ Hemossiderose } & \multicolumn{2}{c}{ Grupo 1 } & & \multicolumn{2}{c}{ Grupo 2 } \\
\cline { 2 - 3 } \cline { 6 - 7 } & $\mathbf{n}$ & $\mathbf{\%}$ & & $\mathbf{N}$ & $\mathbf{\%}$ \\
\hline Leve & 1 & 6,7 & & 15 & 100,0 \\
Moderada / Acentuada & 14 & 93,3 & & 0 & 0,0 \\
\hline TOTAL & $\mathbf{1 5}$ & $\mathbf{1 0 0 , 0}$ & & $\mathbf{1 5}$ & $\mathbf{1 0 0 , 0}$ \\
\hline
\end{tabular}

Nota: $p<0,001$ ( $\mathrm{O}$ valor de $p$ refere-se ao teste exato de Fisher)

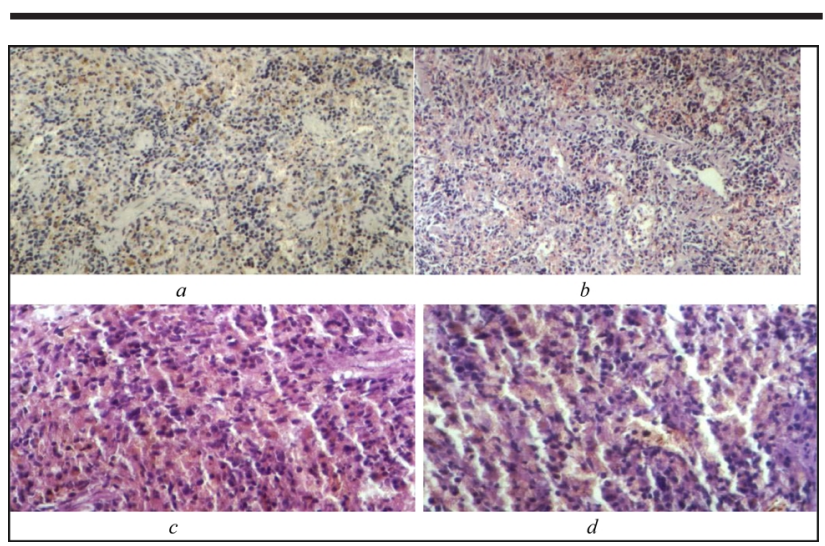

FIGURA 2 - Estudo histológico, por microscopia ótica, dos baços de animais do grupo $1(a$ e $b$ ) e grupo 2 $(c$ e $d)$, nos quais se diagnosticaram estase esplênica associada à hemossiderinose moderada $(a)$; estase sinusoidal de grau moderado da polpa vermelha $(b)$; congestão importante da polpa vermelha (c); na região subcapsular esplênica, a presença de congestão moderada da polpa vermelha $(d)$. Coloração pela hematoxilina-eosina. Aumentos de 200X ( $a$ e $b$ ) e 400X ( $c$ e $d)$.

\section{Discussão}

A incidência de morte dentre os animais do grupo 1 estão de acordo com dados da literatura ${ }^{(10)} \mathrm{e}$ correlacionaram-se com a necrose acentuada dos hepatócitos e da trombose nas veias hepáticas, porta e centro lobular, já que no presente experimento, estes achados apresentaram diferença significantes entre os animais do grupo 1 em comparação aos grupo 2. Esses resultados estão de acordo com outro estudo em ratos, que mostrou a diminuição da agregação plaquetária e leucocitária do edema secundário aos fenômenos de reperfusão e da hiporreatividade de macrófagos e das células de Kupffer hepáticas, fenômenos estes atribuídos à oxigenoterapia hiperbárica e à inibição do óxido nítrico ${ }^{(14)}$. A atenuação das alterações histopatológicas encontradas no grupo 2 e a conseqüente redução significativa da mortalidade pós-operatória parecem relacionar-se à capacidade da oxigenoterapia hiperbárica de reduzir a resposta inflamatória sistêmica após condições clínicas graves e agir de maneira atenuante, criando condições para que os animais tolerassem melhor a congestão hepática grave, secundário a ligadura das veias hepáticas. Em outros estudos verificou-se que o tratamento hiperbárico imediatamente após eventos isquêmicos diminui os fenômenos deletérios secundários à síndrome de reperfusão de forma sistêmica e não apenas local. Sabe-se que em apenas uma hora após o emprego da oxigenoterapia hiperbárica em ratos, ocorreu redução da agregação leucocitária no endotélio venoso, as paredes venosas da musculatura esquelética apresentaram-se livres e bem definidas histologicamente dos fenômenos de reperfusão e houve inibição da isquemia causada pela vasoconstrição microarteriolar, que ocorre após eventos de reperfusão. $\mathrm{O}$ tempo de inicio da oxigenoterapia hiperbárica é importante, 
uma vez que a hiperóxia altera as vias bioquímicas responsáveis pela produção de radicais livres em favor de produtos menos tóxicos aos tecidos, além de reduzir a ativação endotelial, o que diminui a atração dos neutrófilos. Outro mecanismo pelo qual a oxigenoterapia hiperbárica atua sobre os neutrófilos se dá por meio da seqüestração preferencial dos neutrófilos primários em órgãos mais sensíveis a hiperóxia, tais como os pulmões, o que inibe a liberação dessas células na corrente sangüínea ${ }^{(6,8)}$. A oferta de $\mathrm{O}_{2}$ em altas doses diminui a produção da enzima superóxido dismutase, originada durante eventos isquêmicos, sendo responsável pela produção de outros radicais livres e tóxicos para a biologia celular. A estase venosa grave altera o metabolismo celular e os mecanismos de coagulação, sendo as células vermelhas e brancas da microcirculação local os agentes diretamente responsáveis pela produção de radicais livres. Em estudos experimentais concluiu-se que os inibidores do óxido nítrico atenuaram a hipotensão e a hiporreatividade vascular e também reduziram a lesão hepática avaliada por dosagens bioquímicas. Nos fenômenos de isquemia e reperfusão, os genes celulares favorecem a condição inflamatória, que induz a ativação e a agregação leucocitária e sua migração transcapilar e desequilíbrio do óxido nítrico, o que acarreta aumento dos superóxidos. Uma das conseqüências finais desse processo é a redução da resposta vasodilatadora e o aumento da agregação plaquetária, dos fenômenos trombóticos e da necrose muscular. Esses achados foram prevenidos após a administração da L-arginina, substância sabidamente capaz de interferir na produção do óxido nítrico ${ }^{(15,16\}}$. O óxido nítrico é sintetizado a partir de um átomo de nitrogênio terminal guanidino da L-arginina por um processo distinto de classes de enzimas, classificadas como constitutivas (endotelial e neuronal), que induzem a formação de outra enzima denominada óxido nítrico sintetase. As enzimas constitutivas sintetizam e liberam o óxido nítrico dentro de segundos, em resposta a qualquer evento que cause a entrada extracelular súbita de cálcio ou, ainda, a partir da elevação do cálcio intracelular. Já as enzimas induzidas necessitam de algum tempo para se expressarem e, a partir desse momento, produzem óxido nítrico até que ocorra a morte celular ou até que o substrato e os co-fatores para sua produção sejam degradados ${ }^{(17,18)}$. As expressões de genes responsáveis pela síntese do óxido nítrico foram encontradas em hepatócitos e nas células de Kupffer de ratos com hepatopatias induzidas, o que sugere importante papel desse elemento nas afecções hepáticas. Mas apesar da crescente evidência correlacionando o óxido nítrico com várias afecções, inclusive as hepatopatias, sua constatação torna-se difícil devido à sua meia vida que é de poucos segundos ${ }^{(19)}$. Outros autores observaram que a hiperóxia, base fundamental da oxigenoterapia hiperbárica, é um dos agentes capazes de provocar a inibição da produção do óxido nítrico ${ }^{(20)}$. Existem diversas doenças correlacionadas às alterações na capacidade dos vasos sangüíneos em produzir o óxido nítrico, denominadas disfunções endoteliais. Embora ainda não compreendidas completamente, essas disfunções endoteliais estão relacionadas a distúrbios da hemostasia e dos fenômenos trombóticos, secundários aos diversos tipos de lesões celulares ${ }^{(17)}$.
As diferenças significativas apontadas neste estudo em relação à presença de hemossiderose e hemossiderinofagia nos baços dos animais dos grupos $1 \mathrm{e}$ 2 confirmam princípios fisiológicos descritos na literatura. A hemossiderina é um pigmento resultante da degradação da hemoglobina, que contém o elemento ferro em sua constituição e, assim como a ferritina, podem se depositar excessivamente nos tecidos de forma localizada ou sistêmica. A primeira situação é encontrada nas hemorragias, onde se observa a hemossiderina dentro dos macrófagos adjacentes, cerca de 24 a 48 horas após o início do sangramento. A deposição sistêmica de hemossiderina ocorre especialmente nas anemias hemolíticas e em hemotransfussões repetidas ${ }^{(23)}$. Na literatura inglesa foi encontrado apenas um trabalho no qual há relato do uso da oxigenoterapia hiperbárica em seres humanos com isquemia hepática grave, secundária a trombose aguda da artéria hepática, ocorrida em crianças submetidas ao transplante hepático ortotópico. A análise deste grupo com um grupo controle observou diferença significativa em relação a diminuição de eventos hepáticos graves como necroce, colangite e abscessos. Houve ainda diferença significativa no tempo do re-transplante hepático nas crianças submetidas à oxigenoterapia hiperbárica ${ }^{(21)}$. Nos últimos 25 anos, estudou-se muito a importância do oxigênio como nutriente e metabólito ativo, capaz de causar lesão tecidual, mas o emprego de sua dosagem ideal e sua aplicabilidade em várias afecções clínicas fica para os próximos 25 anos. Já a descoberta do óxido nítrico foi um dos maiores achados da Medicina no século XX. O rápido avanço no entendimento dessa molécula fará com que novas estratégias terapêuticas em humanos despontem no horizonte ${ }^{(17 ; 22)}$.

\section{Conclusões}

- A oxigenoterapia hiperbárica em ratos submetidos à ligadura das veias hepáticas

- reduziu a incidência das mortes pós-operatórias desses animais

- atenuou os efeitos deletérios e precoces sobre o fígado e o baço dos animais, observados pela análise histológica

\section{Referências}

1. Bhasin DK, Malhi NJS. Variceal bleeding and portal hypertension: much to learn, much to explore. Endoscopy. 2002;34:119-28.

2. Valla DC. Hepatic vein thrombosis (Budd-Chiari syndrome). Semin Liver Dis. 2002;22(1): 5-14.

3. Janssen HLA, Garcia-Pagan J-C, Ellias E, Mentha G, Hadengue A, Valla D-C. Budd-Chiari syndrome: a review by an expert panel. Hepatology. 2003;38(3):364-71.

4. Xu PQ, Dang XW. Treatment of membranous BuddChiari syndrome: analysis of 480 cases. Hepatobiliary Pancreat Dis Int. 2004;3(1):73-6.

5. Menon KVN, Shah V, Kamath PS. The Budd-Chiari syndrome. N Engl J Med. 2004;350(6):578-85. 
6. Nylander G, Lewis D, Nordstöm H, Larsson J. Reduction of postischemic edema with hyperbaric oxygen. Plast Reconstr Surg. 1985;76(4):596-601.

7. Edwards RJ, Hoopes JE. Effects of hyperbaric oxygen preservation on rat limb replantation: a preliminary report. Ann Plast Surg. 1991;27(1):31-5.

8. Zamboni WA, Roth AC, Russell RC, Graham B, Suchy H, Kucan JO. Morphologic analysis of the microcirculation during reperfusion of ischemic skeletal muscle and the effect of hyperbaric oxygen. Plast Reconstr Surg. 1993;91(6):1110-23.

9. Chen M-F, Chen H-M, Ueng SWN, Shyr M-H. Hyperbaric oxygen pretreatment attenuates reperfusion injury. Liver. 1998;18(2):110-16.

10. Orloff MJ, Daily PO, Girard B. Treatment of Budd-Chiari syndrome due to inferior vena cava occlusion by combined portal and vena caval decompression. Am J Surg. 1992;163:137-43.

11. Gonzáles JFP, Lanuza EDA, Zurita IM, Méndez SM. Portal hypertensive colopathy: histologic appearance of the colonic mucosa. Hepatogastroenterol. 1998;45: 40-3.

12. Grim PS, Lawrence JR G, Allyn B, Eric B. Hyperbaric oxygen therapy. J Am Med Assoc. 1990;263(16):1-11.

13. Costa-Val R, Silva RCO, Nunes TA, Souza TKDP O papel da oxigenoterapia hiperbárica na doença vascular periférica. J Vasc Bras. 2003;2 (3):177-82.

14. Moncada S, Higss EA. Endogenous nitric oxide: physiology, pathology and clinical relevance. Eur J Clin Invest. 1991;21:361-74.
15. Angel MF, Ramasastry SS, Swartz WM, Narayanan K, Kuhns DB, Basford RE, Futrell JW. The critical relationship between free radicals and degrees of ischemia: evidence for tissue intolerance of marginal perfusion. Plast Reconstr Surg. 1988;81(2):233-39.

16. Hammarlund C, Sundberg T. Hyperbaric oxygen reduced size of chronic leg ulcers: a randomized double-blind study. Plast Reconstr Surg. 1994;93(4): 829-33.

17. Makhoul RG, Fields CF, Cassano AD. Nitric oxide and the vascular surgeon. J Vasc Sur. 1999;30(3):569-72.

18. Howe LM, Boothe DM, Slater MR, Boothe HW, Wilkie $\mathrm{S}$. Nitric oxide generation in a rat model of acute portal hypertension. Am J Vasc Res. 2000;61(10):1173-77.

19. Hartleb M, Michielsen PP, Dziurkowska-Marek, A. The role of nitric oxide in portal hypertensive systemic and portal vascular pathology. Acta Gastroenterol Belg.. 1997;60:222-32.

20. Moncada S, Palmer RMJ, Higss EA. Nitric oxide: Physiology, pathology and pharmacology. Pharmacol Rev. 1991;43(2): 109-42.

21. Mazariegos GV, O’Toole K, Mieles LA, Dvorchik I, Meza M, Briassoulis G, Arzate J, Osorio G, Fung JJ, Reyes JJ. Hyperbaric oxygen therapy for hepatic artery thrombosis after liver transplantation in children. Liver Transplant Surg. 1999;5(5):429-36.

22. White MJ, Heckler FR. Oxygen free radicals and wound healing. Clin Plast Surg. 1990;17(3):473-84.

23. Pittella JEH, Barbosa AJA. Pigmentações: calcificações. In: Brasileiro FG. Patologia. 6ed. Rio de Janeiro: Guanabara Koogan; 2000. p.70-80.

\section{Correspondência:}

Tarcizo Afonso Nunes

R. Prof. Estevão Pinto, 637/1104

30220-060 Belo Horizonte-MG

tan@medicina.ufmg.br
Conflito de interesse: nenhum Fonte de financiamento: nenhuma

Recebimento: 12/05/2005

Revisão: $14 / 06 / 2005$

Aprovação: 11/07/2005

\section{Como citar este artigo:}

Costa-Val R, Nunes TA, Oliveira e Silva RC, Souza TKP. Efeitos da oxigenoterapia hiperbárica em ratos submetidos à ligadura das veias hepáticas: avaliação da mortalidade e da histologia do fígado e baço. Acta Cir Bras. [periódico na Internet] 2006 Jan-Feb;21(1). Disponível em URL: http://www.sciwlo.br/acb 